\title{
Irreversible Processes and the Accelerated-Decelerated Phases of the Universe
}

\author{
G. M. Kremer and M. C. N. Teixeira da Silva \\ Departamento de Física, Universidade Federal do Paraná, \\ Caixa Postal 19044, 81531-990 Curitiba, Brazil
}

Received on 16 December, 2003

\begin{abstract}
A model for the Universe is proposed where it is considered as a mixture of scalar and matter fields. The particle production is due to an irreversible transfer of energy from the gravitational field to the matter field and represented by a non-equilibrium pressure. This model can simulate three distinct periods of the Universe: (a) an accelerated epoch where the energy density of the scalar field prevails over that of the matter field, (b) a past decelerated period where the energy density of the matter field becomes larger than the scalar field energy density, and (c) a present acceleration phase where the scalar field energy density overcomes the energy density of the matter field.
\end{abstract}

\section{Introduction}

In order to take into account the irreversible processes during the evolution of the Universe, cosmological models were proposed where the Einstein field equations are combined with the field equations of the thermodynamic theory of irreversible processes. For a homogeneous and isotropic Universe, represented by the Robertson-Walker metric, there exists only one term related to the irreversible processes, namely the non-equilibrium pressure. In Eckart (or firstorder) thermodynamic theory the non-equilibrium pressure is considered as a constitutive quantity which is proportional to the Hubble parameter and whose proportionality factor is the coefficient bulk viscosity (see e.g. the works [1$3]$ ), whereas in extended (second-order or causal) thermodynamic theory the non-equilibrium pressure is supposed to obey an evolution equation (see e.g. the works [4-12]). These theories are known in the literature as viscous cosmological models.

According to cosmological observations one can distinguish three different periods of the Universe with respect to its acceleration field, namely, (a) an early accelerated epoch, (b) a past decelerated period, and (c) a present accelerated phase.

The early acceleration of the Universe, which refers to the inflationary period, can be modeled as a mixture of a scalar field - the so-called inflaton - and a matter field while the non-equilibrium pressure is identified with the particle production due to an irreversible transfer of energy from the gravitational field to the matter field $[9,13,14]$. The inflaton is supposed to have a negative pressure which is the responsible for the acceleration of the early Universe.

The present acceleration of the Universe is also due to a scalar field with a negative pressure, since it has been observed that the energy density of the present Universe is not due to matter or radiation but to an extraordinary non-baryonic matter and energy. Hence, the present Uni- verse can be modeled as a mixture of a matter field with a scalar field which is identified with the dark energy density. If the irreversible processes are taken into account the non-equilibrium pressure, in this case, is the responsible for the transfer of energy between the matter and gravitational fields. There exist at least two candidates in the literature for the dark energy density, namely the quintessence [1518,10] and the Chaplygin gas (see e.g. [11] and the references therein).

The objective of this work is to present a model of the Universe which could describe the distinct periods of the Universe, beginning with an early accelerated epoch, passing through a decelerated period and leading back to an accelerated phase. For that end we model a homogeneous and isotropic Universe as a mixture of two constituents, namely: (a) a scalar field that plays the role of the inflaton at the early accelerated period and of the dark energy at the present accelerated phase; (b) a matter field that represents the particles classically and which are created from the irreversible transfer of energy from the gravitational field to the matter field. Here we show, among other results, that this model can simulate the three distinct periods: the early accelerated, the past decelerated and the present accelerated periods. Units have been chosen so that $c=\hbar=k=1$.

\section{Field Equations}

The equations of state that relate the pressures of the scalar field $p_{\phi}$ and of the matter field $p_{m}$ to their energy densities $\rho_{\phi}$ and $\rho_{m}$ are given by

$$
\left\{\begin{array}{l}
p_{\phi}=w_{\phi} \rho_{\phi}, \quad \text { with } \quad-1 \leq w_{\phi} \leq 0 \\
p_{m}=w_{m} \rho_{m}, \quad \text { with } \quad 0 \leq w_{m} \leq 1
\end{array}\right.
$$

While the equation of state of the matter is the well-known barotropic equation with $w_{m}=0 ; 1 / 3 ; 2 / 3$ representing a pressureless fluid, radiation and non-relativistic matter, respectively, the motivation for the equation of state of the 
scalar field can be found e.g. in the works $[19,20]$ and in the references therein.

If we consider the irreversible processes of particle production in the Universe, the energy-momentum tensor $T^{\mu \nu}$ is written as

$$
T^{\mu \nu}=(\rho+p+\varpi) U^{\mu} U^{\nu}-(p+\varpi) g^{\mu \nu} .
$$

In the above equation $U^{\mu}$ (such that $U^{\mu} U_{\mu}=1$ ) is the fourvelocity and $\varpi$ denotes the non-equilibrium pressure which is the quantity responsible for particle production $[9,13,14]$ during the evolution of the Universe. Moreover, the pressure $p$ and the energy density $\rho$ of the mixture are given by

$$
\rho=\rho_{\phi}+\rho_{m}, \quad p=p_{\phi}+p_{m} .
$$

From the conservation law of the energy-momentum tensor $T_{; \nu}^{\mu \nu}=0$ it follows the balance equation for the energy density of the mixture that - in a comoving frame and by considering the Robertson-Walker metric - reads

$$
\dot{\rho}+3 H(\rho+p+\varpi)=0 .
$$

The quantity $H=\dot{a}(t) / a(t)$ is the Hubble parameter while $a(t)$ denotes the cosmic scale factor and the over-dot refers to a differentiation with respect to time.

The cosmic scale factor is connected with the energy density of the mixture by the Friedmann equation

$$
H^{2}+\frac{k}{a^{2}}=\frac{8 \pi G}{3} \rho,
$$

where $G$ is the gravitational constant and $k$ assumes the values $+1,0,-1$ for closed, flat and open Universes, respectively.

We assume that the scalar field interacts only with itself and is minimally coupled to the gravitational field. In this case, the balance equation for the energy density of the scalar field decouple from the energy density of the mixture (4) and can be written as

$$
\dot{\rho}_{\phi}+3 H\left(\rho_{\phi}+p_{\phi}\right)=0 \text {. }
$$

Equation (6) is used to get from (4) the balance equation for the energy density of the matter field

$$
\dot{\rho}_{m}+3 H\left(\rho_{m}+p_{m}\right)=-3 H \varpi .
$$

From the above equation it is possible to interpret the term $-3 H \varpi$ as the energy density production rate of the matter field (see e.g. [10, 11]).

The relationship between the cosmic scale factor and the energy density of the scalar field can be obtained from the integration of (6) by considering the equation of state given in (1), yielding

$$
\rho_{\phi}=\rho_{\phi}^{0}\left(\frac{a_{0}}{a}\right)^{3\left(w_{\phi}+1\right)} .
$$

In the above equation $\rho_{\phi}^{0}$ is the value of the energy density of the scalar field at $t=0$ (by adjusting clocks) while $a_{0}$ is the corresponding value of the cosmic scale factor.
Now we differentiate the Friedmann equation (5) with respect to time and get the following equation for the time evolution of the cosmic scale factor

$$
\begin{gathered}
\dot{H}+\frac{3}{2}\left(w_{m}+1\right)\left(H^{2}+\frac{k}{a^{2}}\right)=\frac{k}{a^{2}} \\
+4 \pi G\left[\left(w_{m}-w_{\phi}\right) \rho_{\phi}^{0}\left(\frac{a_{0}}{a}\right)^{3\left(w_{\phi}+1\right)}-\varpi\right] .
\end{gathered}
$$

In order to find a solution of (9) for the cosmic scale factor one has to specify how the non-equilibrium pressure $\varpi$ is connected with $a(t)$. Here we assume that $\varpi$ is a variable within the framework of extended (causal or secondorder) thermodynamic theory and write the linearized evolution equation for the non-equilibrium pressure as

$$
\varpi+\tau \dot{\varpi}=-3 \eta H
$$

For the derivation of this equation within the framework of the Boltzmann equation one is referred to [21]. Moreover, the coefficient of bulk viscosity $\eta$ and the characteristic time $\tau$ are assumed to be related to the energy density of the mixture $\rho$ by

$$
\eta=\alpha \rho, \quad \text { with } \quad \tau=\eta / \rho,
$$

where $\alpha$ is a constant (see e.g. [4, 9, 10, 11]).

For the solution of the system of differential equations (9) and (10) we introduce the dimensionless quantities

$$
H \equiv \frac{H}{H_{0}}, \quad t \equiv t H_{0}, \quad a \equiv \frac{a}{a_{0}}, \quad \varpi \equiv \frac{8 \pi G \varpi}{3 H_{0}^{2}},
$$

and the dimensionless coefficients

$$
\alpha \equiv \alpha H_{0}, \quad \chi=H_{0}^{2} a_{0}^{2},
$$

where the index zero denotes the value of the quantity at $t=0$ (by adjusting clocks).

Now the system of differential equations (9) and (10) can be written in terms of the dimensionless quantities (12) and (13) as

$$
\begin{gathered}
\dot{H}+\frac{3}{2}\left(w_{m}+1\right)\left(H^{2}+\frac{k}{\chi a^{2}}\right)=\frac{k}{\chi a^{2}} \\
+\frac{3}{2}\left[\left(w_{m}-w_{\phi}\right) \frac{1+k / \chi}{1+\rho_{m}^{0} / \rho_{\phi}^{0}}\left(\frac{1}{a}\right)^{3\left(w_{\phi}+1\right)}-\varpi\right], \\
\varpi+\alpha \dot{\varpi}=-3 \alpha H\left(H^{2}+\frac{k}{\chi a^{2}}\right) .
\end{gathered}
$$

With respect to the above dimensionless quantities the energy densities of the scalar and matter fields, can be determined from (8) and (5) and read

$$
\frac{\rho_{\phi}}{\rho_{\phi}^{0}}=\left(\frac{1}{a}\right)^{3\left(w_{\phi}+1\right)},
$$

$$
\frac{\rho_{m}}{\rho_{\phi}^{0}}=\frac{1+\rho_{m}^{0} / \rho_{\phi}^{0}}{1+k / \chi}\left(H^{2}+\frac{k}{\chi a^{2}}\right)-\left(\frac{1}{a}\right)^{3\left(w_{\phi}+1\right)}
$$


By specifying initial conditions for $a(t), \dot{a}(t)$ and $\varpi(t)$ at time $t=0$ and values for the parameters $w_{\phi}, w_{m}, \rho_{m}^{0} / \rho_{\phi}^{0}$, $k, \chi$ and $\alpha$ one can determine from the system of differential equations (14) and (15) the time evolution of the cosmic scale factor $a(t)$ and of the non-equilibrium pressure $\varpi(t)$. Once $a(t)$ is a known function of time the energy densities are obtained from (16) and (17). The parameters $w_{\phi}, w_{m}$, $\rho_{m}^{0} / \rho_{\phi}^{0}, k, \chi$ and $\alpha$ have the following interpretation: (a) $w_{\phi}$ and $w_{m}$ represent the ratios between the pressures and the energy densities of the scalar field and matter fields, respectively; (b) $\rho_{m}^{0} / \rho_{\phi}^{0}$ gives the ratio between the initial amount of the energy density of the matter field with respect to that of the scalar field; (c) $k$ and $\chi$ are related with the spacetime geometry; and (d) $\alpha$ is connected with the relevancy of the irreversible processes that correspond to the particle production.

\section{Results and Discussions}

We have solved the system of differential equations (14) and (15) numerically by considering the following initial conditions: $a(0)=1$ for the cosmic scale factor, $H(0)=1$ for the Hubble parameter and $\varpi(0)=0$ for the non-equilibrium pressure. There still remains much freedom to find the solution of the two systems of differential equations, since they do depend on the parameters $w_{\phi}, w_{m}, \rho_{m}^{0} / \rho_{\phi}^{0}, k, \chi$ and $\alpha$. The Figs. 1 and 2 were obtained by choosing: (a) $w_{\phi}=-0.45$ and $w_{m}=1 / 3$ for the ratios between the pressures and the energy densities of the scalar field and of the matter fields, respectively; (b) $\rho_{m}^{0} / \rho_{\phi}^{0}=0$, i.e., a vanishing initial amount of the energy density of matter field since the particles are created through the irreversible process of energy transfer from the gravitational field to the matter field; (c) $\chi=3$ and $k=0,+1,-1$ for the importance of the space-time geometry and (d) $\alpha=0.3$ for the influence of the irreversible processes in the particle production. We call attention to the fact that $w_{m}=1 / 3$ refers to a radiation field and the condition $w_{\phi}=-0.45$ satisfies the restriction for the quintessence $w_{\phi}<-1 / 3$ (see e.g. [20]). Below we shall comment how the change of these parameters affect the solution of the system of differential equations.

We have plotted in Fig. 1 the time evolution of the acceleration field whereas in Fig. 2 it is shown the time evolution of the energy densities for the scalar and matter fields in the cases of closed (straight line), flat (dashed line) and open (dotted line) Universes. These figures follow from the numerical solution of the system of differential equations (14) and (15) for the cosmic scale factor and for the nonequilibrium pressure and from the equations (16) and (17) for the energy densities. One can infer from Figs. 1 and 2 that there exist three distinct periods: (a) an accelerated epoch where the energy density of the scalar field prevails over the matter field, (b) a past decelerated period where the energy density of the matter field becomes more predominant than that of the scalar field, and (c) a present accelerated phase where the scalar field energy density overcomes the

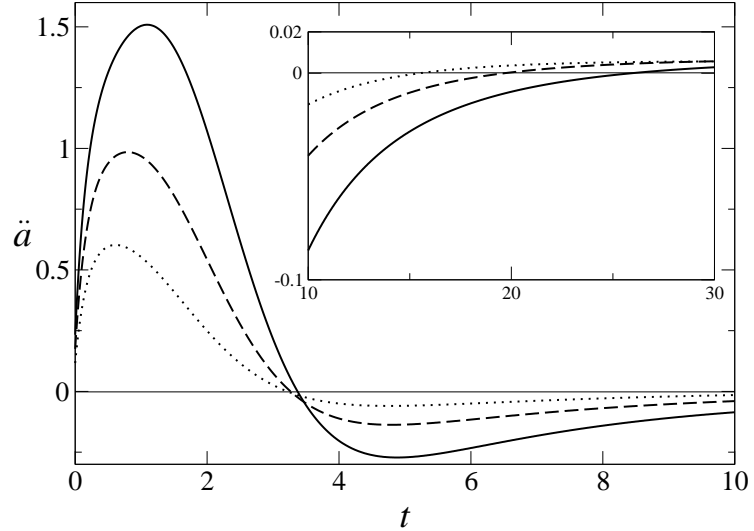

Figure 1. Acceleration $\ddot{a}$ vs time $t$ for closed (straight line), flat (dashed line) and open (dotted line) Universes.

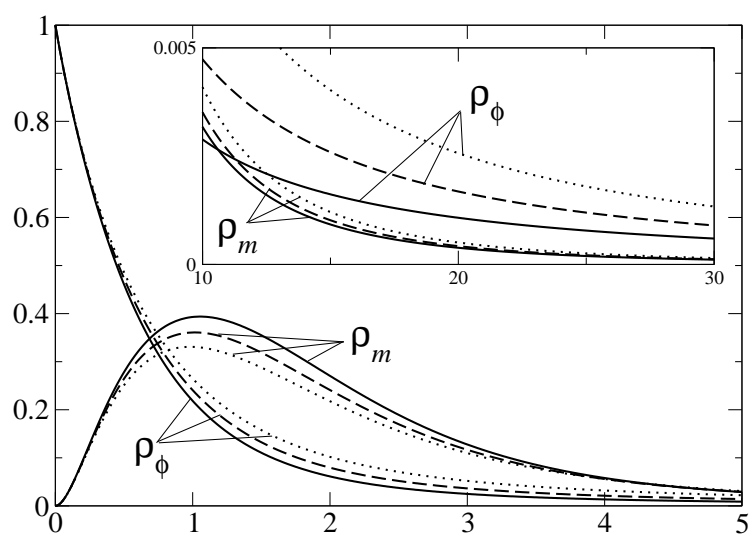

Figure 2. Energy densities of scalar $\rho_{\phi}$ and matter $\rho_{m}$ fields vs time $t$ for closed (straight lines), flat (dashed lines) and open (dotted lines) Universes.

energy density of the matter field. Hence, the transition from the early accelerated period to the decelerated epoch is connected with the transition from a scalar field to a matter field dominated Universe, whereas the transition from the decelerated phase to the present accelerated epoch is connected with the transition from a matter field to a scalar field dominated Universe. In the first accelerated period the closed Universe has the largest value for the acceleration field followed by the flat and the open Universes. One can understand this behavior by recognizing from the equation for the non-equilibrium pressure (15) that $\varpi$ has the largest negative value for the closed Universe followed by the flat and the open Universes which implies that the acceleration, given by

$$
\frac{\ddot{a}}{a}=-\frac{4 \pi G}{3}(\rho+3 p+3 \varpi),
$$

becomes the largest also for the closed Universe. The deceleration is the largest for the closed Universe since more matter is created in a closed Universe (see Fig. 2). This behavior is a direct consequence of the fact that the non-equilibrium pressure is the responsible for the creation of the matter field and it is larger in a closed Universe. The present acceleration is attained first by the open Universe followed by the flat 
and closed Universes since the energy density of the scalar field decays more slowly and lesser matter field is created in the case of the open Universe than the two others.

We have the following remarks concerning the change of the parameters: (a) by decreasing the values of the parameter for the scalar field $w_{\phi}$ the energy density of the scalar field decays more slowly (see equation (16)) so that the initial accelerated period increases and the deceleration period decreases; (b) when the value of the parameter $w_{m}$ for the matter field is increased in the interval between radiation and non-relativistic matter $1 / 3 \leq w_{m} \leq 2 / 3$ the energy density of the matter field decreases (see equation (7)) so that there exists a smaller decelerated period; (c) when $w_{m}$ is decreased in the interval between dust and radiation $0 \leq w_{m} \leq 1 / 3$ this behavior reverses; (d) by decreasing the value of the parameter $\chi$ - which refers to the importance of the space-time geometry - the period of past deceleration becomes larger for the closed Universe and smaller for the open Universe, since for the former case the nonequilibrium pressure becomes more negative than that of the latter (see equation (15)) ; (e) by increasing the value of the parameter $\alpha$ - which is liable for the significance of irreversible processes - the period of past deceleration becomes larger for the closed Universe and smaller for the open Universe, which confirms that the irreversible processes are connected with the particle production and that the energy density of the matter field answer for the decelerated period. From the above remarks we infer that the behavior of the solutions found here for the acceleration field and for the scalar and matter energy densities is also valid by changing the values of the parameters $w_{\phi}, w_{m}, \rho_{m}^{0} / \rho_{\phi}^{0}, k, \chi$ and $\alpha$ within specified ranges.

\section{References}

[1] G. L. Murphy, Phys. Rev. D 8, 4231 (1973).
[2] Ø. Grøn, Astrophys. Space Sci. 173, 191 (1990).

[3] G. M. Kremer and F. P. Devecchi, Phys. Rev. D 65, 083515 (2002).

[4] V. A. Belinskií, E. S. Nikomarov, and I. M. Khalatnikov, Sov. Phys. JETP 50, 213 (1979).

[5] V. Romano and D. Pavón, Phys. Rev. D 47, 1396 (1993).

[6] L. P. Chimento and A. S. Jakubi, Class. Quant. Grav. 10, 2047 (1993).

[7] A. A. Coley and R. J. van den Hoogen, Class. Quant. Grav. 12, 1977 (1995).

[8] W. Zimdahl, Phys. Rev. D 61, 083511 (2000).

[9] G. M. Kremer and F. P. Devecchi, Phys. Rev. D 66, 063503 (2002).

[10] G. M. Kremer and F. P. Devecchi, Phys. Rev. D 67, 047301 (2003).

[11] G. M. Kremer, Gen. Relat. Grav. 35, 1459 (2003).

[12] G. M. Kremer, Phys. Rev. D 68, 123507 (2003).

[13] I. Prigogine, J. Geheniau, E. Gunzig, and P. Nardone, Gen. Relat. Grav. 21, 767 (1989).

[14] W. Zimdahl, Phys. Rev. D 57, 2245 (1998).

[15] R. R. Caldwell, R. Dave, and P. J. Steinhardt Phys. Rev. Lett 80, 1582 (1998).

[16] I. Zlatev, L. Wang, and P. J. Steinhardt Phys. Rev. Lett 82, 896 (1999).

[17] W. Zimdahl, D. Pavón, and L. P. Chimento, Phys. Lett. B 521, 133 (2001).

[18] W. Zimdahl, A. B. Balakin, D. J. Schwarz, and D. Pavón, Grav. Cosmol. 8, 158 (2002).

[19] B. Ratra and P. J. E. Peebles, Phys. Rev. D 37, 3406 (1988).

[20] P. J. E. Peebles and B. Ratra, Rev. Mod. Phys. 75, 559 (2003).

[21] C. Cercignani and G. M. Kremer, The Relativistic Boltzmann Equation: Theory and Applications, (Birkhäuser, Basel, 2002). 